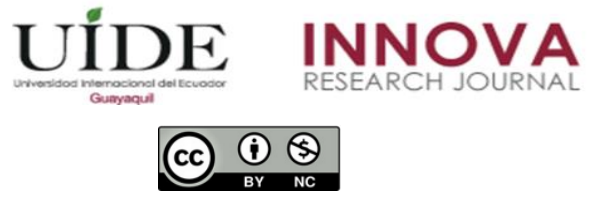

INNOVA Research Journal, ISSN 2477-9024

(Enero - Abril 2021). Vol. 6, No.1 pp. 1-17

DOI: https://doi.org/10.33890/innova.v6.n1.2021.1393

URL: http://revistas.uide.edu.ec/index.php/innova/index

Correo: innova@uide.edu.ec

\title{
Liderazgo transformacional y transaccional en la autoeficacia emprendedora del turismo comunitario amazónico, caso provincia de Pastaza
}

\section{Transformational and transactional leadership in the entrepreneurial self- efficacy of Amazonian community tourism, case of Pastaza province}

Luis Oswaldo Manosalvas Vaca

(D) https://orcid.org/0000-0002-4659-8090

Universidad Estatal Amazónica, Ecuador

Angélica Aracely Tobanda Barragán

(iD) https://orcid.org/0000-0003-3906-862X

Investigador Independiente, Ecuador

Carlos Aníbal Manosalvas Vaca

(D) https://orcid.org/0000-0002-7521-069X

Universidad Estatal Amazónica, Ecuador

Deisy Valeria Quevedo Amay

(D) https://orcid.org/0000-0002-3782-6354

Universidad Estatal Amazónica, Ecuador

Autor para correspondencia: Imanosalvasvaca@ hotmail.com; aracelytobanda2@ hotmail.com; cmanosalvas@uea.edu.ec; dv.quevedoa@uea.edu.ec

Fecha de recepción: 06 de abril de 2020 - Fecha de aceptación: 07 de agosto de 2020

\section{Resumen}

La presente investigación tiene como objetivo analizar la relación del Liderazgo transformacional y transaccional con la Autoeficacia emprendedora en una comunidad indígena ubicada en Amazonía ecuatoriana, Provincia de Pastaza, donde se desarrolló una evaluación de las características demográficas de la muestra y un análisis descriptivo de las variables de estudio, para analizar la variable liderazgo se utilizó el cuestionario multifactorial de liderazgo (Multifactor Leadership Questionnaire (MLQ), y el instrumento de autoeficacia emprendedora desarrollado por De Noble et al. (1999). Además, se midió la confiabilidad de los instrumentos con un análisis de Cronbach y mediante la correlación de Pearson se determinó que el liderazgo transformacional se relaciona de manera significativa con la autoeficacia emprendedora, sin embargo, el liderazgo transaccional no se relaciona con la autoeficacia emprendedora; los resultados indicaron que la actividad emprendedora de la comunidad tiene una influencia de carácter transformacional a través de sus emprendedores líderes, quienes apoyan el desarrollo emprendedor considerando el avance personal de los miembros, sin embargo, no se ha identificado una relación de un proceso de transacción o recompensa en las actividades de 
emprendimiento, esto permite generar posibles investigaciones que analicen futuros comportamientos de emprendimiento con respecto a procedimientos transaccionales $\mathrm{y}$ transformacionales de liderazgo en el emprendimientos en diversos contextos similares.

Palabras claves: liderazgo; transformacional; transaccional; autoeficacia; emprendimiento.

\begin{abstract}
The present research aims to analyze the relationship between transformational and transactional Leadership with entrepreneurial self-efficacy in an indigenous community located in the Ecuadorian Amazon, Pastaza Province, where an evaluation of the demographic characteristics of the sample and a descriptive analysis of the variables were developed, study, to analyze the leadership variable, the multifactorial leadership questionnaire (Multifactor Leadership Questionnaire (MLQ), and the entrepreneurial self-efficacy instrument developed by De Noble et al. (1999) were used. In addition, the reliability of the instruments was measured with A Cronbach analysis and through Pearson's correlation it was determined that transformational leadership is significantly related to entrepreneurial self-efficacy, however, transactional leadership is not related to entrepreneurial self-efficacy; the results indicated that the entrepreneurial activity of the community has a transformational influence through its leading entrepreneurs, who support entrepreneurial development considering the personal advancement of the members, however, a relationship of a transaction or reward process has not been identified in entrepreneurial activities, this allows generating possible Research that analyzes future entrepreneurial behaviors with respect to transactional and transformational leadership procedures in entrepreneurship in various similar contexts.
\end{abstract}

Keywords: leadership; transformational; transactional; self-efficacy; entrepreneurship.

\title{
Introducción
}

En la actualidad las sociedades comunitarias se caracterizan por ser más demandantes en su entorno social, en ellas su territorio y cultura son la base de su economía, la cual se desarrolla de mejor manera con la creación de empresas que dinamizan la misma (Ledesma Almache, 2016). En las comunidades la función del liderazgo en el emprendimiento es relevante para respaldar y desarrollar la innovación en la creación de productos, evitando que los mismos lleguen a estar limitados en el tiempo (Salazar, Vargas, y Salazar, 2017). Lasio, Caicedo, Ordeñana, Samaniego y Zambrano (2016) en su investigación realizada en Ecuador para Global Entrepreneurship Monitor, indicaron que los emprendedores juegan un rol crítico en el desarrollo y bienestar de las sociedades; pues al estudiar las actitudes, actividades y aspiraciones relacionadas a los emprendimientos se encontró que debe existir una serie de aspectos relacionados con factores internos y externos que impulsen su desarrollo (Orozco Dussán, 2014). El liderazgo y las habilidades emprendedoras de los individuos es un tema de investigación que ha cobrado mucha importancia durante los últimos años. (Sopó, Salazar, Guzmán y Vera, 2017); sin embargo, Fernández y Quinteros (2017) señalaron que la teoría del liderazgo transaccional y transformacional genera un conocimiento más profundo sobre los aspectos fundamentales que tienen los líderes para confrontar los cambios frecuentes de un mundo globalizado, las investigaciones han indicado que un liderazgo efectivo evidencia la combinación de ambos estilos de liderazgo (Avolio, Bass yJung, 1999). 
Fernández y Quinteros (2017) indicaron que el liderazgo transformacional incrementa la actitud de compromiso y la motivación, en cambio, el liderazgo transaccional se direcciona hacia generar recompensas entre el líder y los miembros de la organización, como resultado del trabajo realizado, estas relaciones pueden influir en diversos aspectos dentro de las organizaciones y también en entornos determinados por emprendimientos (Orozco Dussán, 2014). Con estos antecedentes podemos verificar que la relación entre liderazgo transformacional y transaccional con la percepción de las habilidades de emprendimiento en el sector turístico es de gran importancia y al carecer de evidencia de éstas dos variables en localidades en vías de desarrollo, específicamente en comunidades Amazónicas, se hace relevante la investigación. Los pueblos indígenas Amazónicos se están involucrando cada vez más en emprendimientos turísticos en todo el mundo, pues esto, en gran medida es una respuesta al crecimiento poblacional en áreas de vida para muchas comunidades; sin embargo, para que dichas empresas se consideren exitosas los pueblos indígenas deben ser capaces de tomar el control a pesar de las fuerzas que a menudo trabajan en contra de ellos (Peredo, Ordóñez y Belohrad, 2015). Las comunidades que desarrollan un buen liderazgo transaccional y transformacional, y fomentan las habilidades de sus miembros pueden participar en actividades económicas como el turismo y pueden beneficiarse de ellas. Los actores de la actividad emprendedora actúan en base a juicios de valor, ya sea de manera individual o en grupos de asociaciones de emprendimiento (Orozco Dussán, 2014). Por esta razón. Mendoza y Prideaux (2017) indicaron que las comunidades para desarrollar negocios sostenibles requieren el apoyo de sus líderes comunitarios y de potenciar las capacidades propias de cada uno de los habitantes que la conforman.

\section{Liderazgo}

El entorno social se ha desarrollado en base actividades de liderazgo, lo cual ha permitido generar diversos intereses de investigación en varias áreas de aplicación (Antares, 2013). Según Gómez, Quero, López, Medina y Pérez (2016) el líder es quien tiene la habilidad de transformar y generar compromiso con su persona, las organizaciones, el entorno familiar y comunitario. De igual forma, se debe separar las funciones de quien cumple su rol como jefe y aquel que tiene las habilidades de ser un líder (Munch, 2011).

Por otro lado, el hecho de ser líder no implica que los resultados de su gestión sean sobresalientes ni tampoco perdurables (Vadillo Bueno, 2011). La actividad relevante del liderazgo, no necesariamente se manifiesta en los resultados, es en el proceso mismo donde se evidencia los valores y conductas que pueden influir y ser un ejemplo como modelo de aplicación atractivo y de imitación (Corporate Excellente, 2014). Un líder crea compromiso y desarrolla la creatividad, de tal manera, que puede manifestarse en base al comportamiento como base fundamental de las prioridades y principios de las personas (Naranjo, 2015). El liderazgo pone de manifiesto el guiar como actividad principal las organizaciones y conjuntamente con el talento humano el cumplimiento de objetivos estratégicos; la falta de un liderazgo limita el desarrollo del proceso administrativo en todas sus etapas y elementos, lo cual abre posibilidades de riesgo para que los emprendimientos puedan fracasar (Noriega, 2008).

\section{Liderazgo Transformacional}


El liderazgo transformacional produce cambios en el estilo de comportamiento de las personas, lo cual influye en las organizaciones, se reinventa la forma de valorar y percibir el entorno, se desarrolla una comunicación más fluida, se genera inspiración, compromiso, permitiendo una mayor motivación y confianza con los subalternos, incrementando la efectividad en el desarrollo organizacional (Burns, 1978; Hermosilla, Amutio, Da Costa, y Páez, 2016). El liderazgo transformacional actúa sobre las actividades intangibles, creando relaciones de compromiso en los integrantes de la organización empresarial (Varela, 2010).

El líder transformacional asume riesgos, tiene una visión más objetiva para reconocer oportunidades, tiene apertura a crear experiencia y adquirir nuevo aprendizaje, transmite inspiración e interés en las actividades que realiza hacia sus seguidores para conseguir las metas organizacionales (Fernández y Quintero, 2017). En resumen, el liderazgo transformacional: (1) satisface necesidades de los miembros de la organización (2) conoce lo que necesita el equipo de trabajo, (3) mejora la contribución y desempeño laboral y (4) el líder se considera como un miembro del equipo de trabajo (Cruz-Ortiz, Salanova, y Martínez, 2013; Llorens, Salanova, y Losilla, 2009; Nielsen, 2014). Este tipo de liderazgo se considera como un valioso recurso de carácter social que mejora los niveles de desempeño en las empresas (Olvera, Llorens, Acosta y Salanova, 2017)

Según Bass y Avolio (1994) el liderazgo transformacional tiene cuatro dimensiones:

- Influencia Idealizada. - creando una alta conducta ética y moral, lo cual determina una lealtad hacia el líder.

- Motivación Inspiradora. - para lograr las metas propuestas en base a los valores presentes.

- Estimulación Intelectual. - estimulando la innovación en el entorno que se desarrolla.

- Consideración Individual. - identificando el comportamiento de los actores del grupo de trabajo para desarrollar habilidades de liderazgo.

\section{Liderazgo Transaccional}

Bass (1985), define al liderazgo transaccional el que está basado en el intercambio de recompensas entre el líder y los miembros de su equipo. En el liderazgo transaccional se evidencia la sanción o recompensa del trabajador en base al desempeño o rendimiento, este tipo de liderazgo se ajusta a los procedimientos normales de las tareas de trabajo, sin considerar un desarrollo a futuro de la actividad empresarial (Contreras y Barbosa, 2013).

El liderazgo transaccional se establece mediante un carácter legal y burocrático en las empresas, en donde el uso de la recompensa es el mecanismo para favorecer la motivación laboral (Durán y Castañeda, 2015). Los miembros de una organización ofrecen sus servicios de trabajo y como resultado de esta actividad reciben una compensación económica facilitando en los trabajadores los recursos que permitan los objetivos personales y organizacionales. La recompensa de pago es un sistema en que ambas partes ganan por un esfuerzo realizado (Guanilo, 2017). 
Bass y Avolio (2000) señalaron que el liderazgo transaccional tiene las siguientes dimensiones:

- Recompensa contingente. - incentivando el reconocimiento de las metas obtenidas.

- Gestión por excepción activa.- en una normativa de la organización se identifica los errores y posibles desviaciones para implementar soluciones.

- Gestión por excepción pasiva. - actuaciones del líder cuando surgen diversos problemas en la empresa.

- Laissez-faire. - no se evidencia una responsabilidad por parte del líder, se conoce como un no liderazgo.

El liderazgo transaccional es efectivo en el entorno empresarial tradicional, para generar estrategias que permitan cumplir las metas organizacionales (Contreras y Barbosa. 2013). El liderazgo transformacional y transaccional trabajan de manera conjunta, mientras que el liderazgo transformacional intenta cambiar la cultura organizacional, el liderazgo transaccional trabaja en la cultura existente (Fernández y Quintero, 2017).

Para medir los estilos de liderazgo en las investigaciones relevantes se utiliza el instrumento Multifactor Leadership Questionnaire (MLQ) (Bass y Avolio, 1994). Este instrumento ha pasado por varias modificaciones y se han creado algunas versiones para su aplicabilidad, en la actualidad existe una versión del MLQ validada en España que se utiliza en investigaciones de habla hispana (Molero F., 1995).

\section{Liderazgo en comunidades}

Las comunidades corresponden a grupos de personas que comparten competencias e intereses comunes que interactúan constantemente para cumplir un proyecto común o para resolver problemas compartidos por los miembros; adoptando así algunos patrones específicos de organización interna donde algunos de sus miembros obtienen un estado de liderazgo; los cuales contribuyen al avance cognitivo de la comunidad de práctica al proporcionar a los miembros una visión consistente y coherente de sus objetivos (Muller, 2016). Whiterford y Pados (2015) afirmaron que la apertura del liderazgo y la construcción de habilidades individuales es la clave para desarrollar verdaderos líderes comunitarios y regionales que sean parte integral de la misma y que verdaderamente sean comprometidos a crear un cambio social sostenible.

El sistema de liderazgo tradicional en las comunidades, normalmente es temporal y fragmentario, el cual es repartido entre familias, bandas, linajes y varias asociaciones. Al ser fragmentado el liderazgo se vuelve débil, pues las gestiones de recursos importantes que responden a solucionar amenazas inmediatas para toda la comunidad no son atendidas de manera primordial por los diferentes grupos que mantienen dicho poder (Torres, 2015). Sin embargo, dichas comunidades han encontrado maneras de hacer que este liderazgo funcione, volviendo a éste un facilitador de alianzas para gestionar recursos en momentos de crisis, bajo una organización que se basa en una red social con valores como la colaboración y reciprocidad a lo interno de la comunidad (Torres, 2015). Tal proceso da lugar a la aparición de líderes que son considerados como dispositivos importantes que contribuyen a la coordinación interna de 
comunidades, es decir, los líderes son miembros específicos que se caracterizan por niveles más altos de actividad en la comunidad (Muller, 2016).

\section{Autoeficacia emprendedora}

La autoeficacia emprendedora es el constructo más viable que ha permitido comprender el comportamiento de actividades de emprendimiento en diversas áreas. Según las investigaciones quienes poseen altos niveles de autoeficacia emprendedora usan sus habilidades y esfuerzos para desarrollar y mantener los emprendimientos durante el tiempo (López, Flores, y Bojórquez, 2016). La autoeficacia emprendedora evalúa la percepción de un emprendedor sobre las capacidades y habilidades para realizar diversas acciones cuando se desarrolla el emprendimiento, en resumen, se refiere a las creencias propias del emprendedor para ejecutar las situaciones que conllevan un emprendimiento (Krueger y Carsrud, 1993). Las creencias propias en las habilidades de emprendimiento tienen un impacto positivo en la manera de pensar y comportarse de los emprendedores, se ha considerado esta variable como un precursor de la intención de emprendimiento (Bandura, 1999).

La autoeficacia se ha relacionado con diversos constructos que tratan de explicar el comportamiento emprendedor, generando respuestas relacionadas hacia actitudes que explican factores que determinan la actividad emprendedora, manteniéndose en un mercado competitivo en el largo plazo, integrando aspectos relacionados a la innovación y la creatividad (López, Flores, y Bojórquez, 2016). La autoeficacia emprendedora es una característica distintiva del emprendedor y las comunidades pueden trabajar para crear un entorno que mejore la misma al hacer que los recursos estén disponibles y sean visibles, publicitar los éxitos empresariales, aumentar la diversidad de oportunidades y evitar políticas que creen obstáculos reales o percibidos. En cualquier caso, lo que sí queda claro es que la autoeficacia percibida afecta el comportamiento no sólo directamente, sino también como variable interviniente y mediadora, condicionando aspiraciones, expectativas, predisposiciones y hasta la evaluación de posibilidades de éxito/fracaso en el medio social.

Diversas investigaciones han reportado factores propios que caracterizan el comportamiento emprendedor y que se relacionan con habilidades y competencias emprendedoras, entre las principales se encuentran la motivación, el liderazgo, la personalidad, la toma de riesgos, la autoconfianza, la creatividad; de igual manera, se ha reportado factores relacionados al ambiente comunitario, social, organizacional. De igual forma, existen atributos que integran la creación de nuevos emprendimientos como: los marcos de referencia familiar y social, el género, la edad, la ubicación geográfica, la educación recibida, el grado de conocimiento en una temática específica, la experiencia laboral (Palma, 2011). En consecuencia, se puede observar que las habilidades de emprendimiento son una vía relevante para el crecimiento y desarrollo de un país. (Pérez, Fernández, Ubierna, y Arranz, 2016) y es ahí donde radica la creciente importancia en conocer cómo se desarrollan las habilidades emprendedoras, las cuales crean actividad económica en las regiones mediante el desarrollo laboral y empresarial (Xu, 2014). Por esta razón, los gobiernos han invertido ingentes cantidades de recursos para desarrollar estas habilidades en sus ciudadanos. (Pérez, Fernández, Ubierna, y Arranz, 2016), pues las mismas están influenciadas por las condiciones económicas, sociales, culturales y políticas. (Lasio, Caicedo, Ordeñana, Samaniego, y Zambrano, 2016) 


\section{Autoeficacia emprendedora en el desarrollo turístico de comunidades}

En la actualidad el factor humano es el elemento clave que impulsa, diferencia y genera un valor en su entorno, capaz de perdurar en el tiempo. Para su gestión efectiva, se deben establecer metas, donde el individuo tengan definido un camino que le permita poner en práctica sus habilidades de emprendimiento; por lo que resulta primordial entonces identificar y potencializar al máximo sus capacidades (Murillo, González, y Perdomo, 2010).

Las comunidades no son ajenas a esta situación, pues a nivel Ecuador el sector turístico se está convirtiendo en la primera apuesta productiva del país como referencia de la economía, por este motivo se debe fomentar el desarrollo del turismo, mediante políticas encaminadas al fortalecimiento del turismo comunitario, tomando en consideración el hecho de que las comunidades se encuentran ubicadas en territorio amazónico en la provincia de Pastaza (Marín, ,Manjarrez, Ortega, Sablón, y Romero, 2017).

El impacto del sector turístico sobre el crecimiento regional y territorial, podría estar determinado por aspectos externos como internos, al igual que por las capacidades de cada emprendedor (Durán y Arias, 2016). La creación de empresas puede estar influenciado por el conocimientos de las creencias propias del emprendedor en sus habilidades (Manosalvas, 2017). Esto ha potenciado los esfuerzos para desarrollar las habilidades de emprendimiento que generen personas capaces de crear iniciativas sostenibles en el tiempo. (Pérez et al, 2016) En Ecuador se ha evidenciado que la actividad turística comunitaria potencializa la creación de estrategias sociales, permitiendo la creación de empleos y crecimiento económico para las culturas tradicionales que se enmarcan en un territorio (Ibarra, Tandazo, Alemán, Bravo y Caballero). Es importante el análisis de los comportamientos emprendedores desde una percepción de la sociedad, al igual que desde la misma comunidad con los propios actores del emprendimiento (Álvarez, Noguera, y Urbano, 2012)

Las investigaciones han reportado que no existe una diferencia significativa en lo que corresponde a género, sin embargo, también se considera que ambientes donde existe la predominancia de un género determinado en los emprendimientos crea una tendencia hacia el segmento masculino o femenino que lo representa (Fischer, Reuber, y Dyke, 1993). Se debe considerar, aunque existen diferencias entre mujeres y hombres, la percepción de la sociedad como de la comunidad en las habilidades de emprendimiento, es un indicativo del éxito emprendedor (Álvarez, et al, 2012). Por otro lado, el contexto cultural en las comunidades determina que tan proclives son al emprendimiento en comparación a otras, (Liñán y Chen, 2009), por lo que Murillo, et al. (2010) indicaron que deben existir estrategias focalizadas a la transformación de la confianza de cada individuo y la responsabilidad social que implica la misma, generando impacto en la gestión de emprendimientos turísticos, especialmente en los que se encuentran en un contexto poco estudiado como son las comunidades.

\section{Liderazgo en la Autoeficacia emprendedora}

La sociedad actual tiene grandes desafíos a nivel individual y colectivo. Es necesario que se generen unas mínimas cualidades para las personas que lideran o puedan liderar emprendimientos en el futuro, porque sus decisiones influirán en el progreso personal pero

Esta obra se comparte bajo la licencia Creative Common Atribución-No Comercial 4.0 International (CC BY-NC 4.0) Revista de la Universidad Internacional del Ecuador. URL: https://www.uide.edu.ec/ 
también de toda la ciudadanía. Somos conscientes de que personas que ocupan posiciones significativas e influyentes son líderes natos; pero es necesario indagar sobre las habilidades necesarias de cómo ser un buen líder (Maldonado, Castro, y Franco, 2017). La relación del liderazgo y la autoeficacia emprendedora se ha evaluado desde diferentes perspectivas, encontrando resultados que relacionan tanto aspectos educativos como demográficos (Sopó, Salazar, Guzmán, y Vera, 2017). Existe la necesidad de conocer la capacidad de ejercer influencia y demostrar las mejores características que mantengan a los mejores talentos en una organización empresarial (Spinelli y Adams, 2012). Kuratko (2007) indicó que el liderazgo permite desarrollar las habilidades de emprendimiento e innovación. Peters (2005) señaló que el liderazgo también puede influir en la motivación empresarial para alcanzar varios objetivos El liderazgo tiene un papel relevante en la autoeficacia emprendedora y por consiguiente en el desempeño empresarial lo cual debe tener un equilibrio con los recursos disponibles de la empresa. (Sopó, et al. 2017).

\section{Materiales y Métodos}

La investigación fue desarrollada mediante una ruta cuantitativa, con un paradigma deductivo, en búsqueda de la relación del liderazgo transaccional y transformacional con la autoeficacia emprendedora, se estableció en un horizonte transversal. Se comenzó seleccionando una muestra ubicada en la Provincia de Pastaza de la región Amazónica del Ecuador, luego se diseñó un análisis descriptivo para identificar datos demográficos como edad, género, localización geográfica y las variables de interés: liderazgo transformacional y transaccional, autoeficacia emprendedora en la muestra analizada. Luego, se procedió a realizar un análisis de correlación bivariada de Pearson entre las variables liderazgo transaccional y transformacional con la autoeficacia emprendedora. La investigación fue desarrollada mediante un trabajo de campo, con encuestas previamente validadas sobre liderazgo y autoeficacia emprendedora. La selección de la muestra se realizó mediante un muestreo probabilístico aleatorio simple lo cual permitió seleccionar los casos que cumplen con una condición en general (Mendieta, 2015), la cual fue tener algún emprendimiento dentro de la comunidad, teniendo como resultado 51 emprendedores que cumplen con esta característica para la inclusión en la muestra.

Se utilizaron instrumentos de medición que fueron sometidos a un Análisis de Confiabilidad de Cronbach:

Cuestionario Multifactorial de liderazgo (Multifactor Leadership Questionnaire (MLQ). Que permitió definir la existencia de dos tipos de liderazgo: el transaccional y el transformacional; además, de posibilitar la realización de comparaciones transculturales (Molero et al, 2010).

El instrumento de autoeficacia emprendedora validado por De Noble et al. (1999) que mide la creencia en las propias habilidades de emprendimiento en la muestra aplicada. 


\section{Resultados}

\section{Confiabilidad de instrumentos de medición}

Se realizó el análisis de confiabilidad de Cronbach, hacia los dos instrumentos considerando que son dos encuestas diferentes que no pueden ser valorados en una sola agrupación.

\section{Tabla 1}

Alfa de Cronbach Multifactorial de liderazgo (Multifactor Leadership Questionnaire MLQ)

\begin{tabular}{llc}
\hline Estadísticos de confiabilidad & \\
\hline Alfa de Cronbach & Alfa de Cronbach basada en los elementos tipificados & N de elementos \\
\hline, 879 &, 911 & 45 \\
\hline
\end{tabular}

El resultado de confiabilidad del MLQ indicó un valor de .879 , lo cual indica una consistencia interna buena (Frías, 2014).

\section{Tabla 2}

Alfa de Cronbach Autoeficacia emprendedora

\begin{tabular}{llc}
\hline Estadísticos de fiabilidad & \\
\hline Alfa de Cronbach & Alfa de Cronbach basada en los elementos tipificados & N de elementos \\
\hline, 947 &, 948 & 23 \\
\hline
\end{tabular}

El coeficiente de confiabilidad del instrumento autoeficacia emprendedora dio un valor de .948, que representa una consistencia interna excelente (Frías, 2014).

\section{Análisis de datos demográficos}

\section{Tabla 3}

Edad de la muestra

\begin{tabular}{llll}
\hline & & Frecuencia & Porcentaje \\
\hline \multirow{3}{*}{ Válidos } & De 18 a 25 años & 19 & 37,3 \\
\cline { 2 - 4 } & De 26 a 35 años & 19 & 37,3 \\
\cline { 2 - 4 } & De 36 a 45 años & 8 & 15,7 \\
\cline { 2 - 4 } & De 46 a 55 años & 4 & 7,8 \\
\cline { 2 - 4 } & De 56 a más años & 1 & 2,0 \\
\cline { 2 - 4 } & Total & 51 & 100,0 \\
\hline
\end{tabular}


La edad de la muestra, permite inferir una población adulta joven ya que se encuentra en un rango de 18 a 45 años.

\section{Tabla 4}

Género de la muestra

\begin{tabular}{llll}
\hline & & Frecuencia & Porcentaje \\
\hline \multirow{2}{*}{ Válidos } & Masculino & 21 & 41,2 \\
\cline { 2 - 4 } & Femenino & 30 & 58,8 \\
\cline { 2 - 4 } & Total & 51 & 100,0 \\
\hline
\end{tabular}

La muestra indica que existe una predominancia del género femenino, lo cual a futuro también permite desarrollar investigaciones sobre la influencia del género en el liderazgo y el emprendimiento.

\section{Tabla 5}

Nivel de estudios

\begin{tabular}{llll}
\hline & & Frecuencia & Porcentaje \\
\hline \multirow{4}{*}{ Válidos } & Bachiller & 37 & 72,5 \\
\cline { 2 - 4 } & Cursando la Universidad & 11 & 21,6 \\
\cline { 2 - 4 } & Título de Tercer Nivel & 3 & 5,9 \\
\cline { 2 - 4 } & Total & 51 & 100,0 \\
\hline
\end{tabular}

La mayoría de los emprendedores tienen una educación de tercer nivel, lo cual podría inferir un emprendimiento por necesidad antes que por oportunidad, pero también podría indicar que la actitud emprendedora no necesariamente depende de una educación más formal.

\section{Tabla 6}

Autodefinición étnica

\begin{tabular}{llll}
\hline & & Frecuencia & Porcentaje \\
\hline Válidos & Shuar & 10 & 19,6 \\
\cline { 2 - 4 } & Achuar & 11 & 21,6 \\
\cline { 2 - 4 } & Kichwa & 19 & 37,3 \\
\cline { 2 - 4 } & Zápara & 4 & 7,8 \\
\cline { 2 - 4 } & Huaorani & 3 & 5,9 \\
\cline { 2 - 4 } & Mestizo & 4 & 7,8 \\
\cline { 2 - 4 } & Total & 51 & 100,0 \\
\hline
\end{tabular}


Se identificaron seis autodefiniciones éticas en las que prevalecen los Shuar, Achuar, y Kichwa.

\section{Análisis descriptivo de las variables Liderazgo transformacional y transaccional}

\section{Tabla 7}

Dimensiones del constructo Liderazgo transformacional

\begin{tabular}{|c|c|c|}
\hline Dimensión & Resultado de Análisis & Interpretación \\
\hline $\begin{array}{l}\text { Influencia idealizada } \\
\text { (atributo) }\end{array}$ & Más del 75\% desfavorable & $\begin{array}{l}\text { El liderazgo no se considera } \\
\text { como un modelo a seguir, } \\
\text { porque no inspira confianza }\end{array}$ \\
\hline $\begin{array}{l}\text { Influencia idealizada } \\
\text { (comportamiento) }\end{array}$ & Más del 70\% desfavorable & $\begin{array}{l}\text { No se evidencia un } \\
\text { comportamiento de liderazgo } \\
\text { ético y moral. }\end{array}$ \\
\hline Motivación inspiradora & Más del 65\% desfavorable & $\begin{array}{l}\text { No se reconoce una meta a } \\
\text { conseguir }\end{array}$ \\
\hline Estimulación intelectual & Más del $70 \%$ desfavorable & $\begin{array}{l}\text { El liderazgo no potencializa la } \\
\text { creatividad y la innovación }\end{array}$ \\
\hline $\begin{array}{l}\text { Consideración } \\
\text { Individualizada }\end{array}$ & Más del 85\% desfavorable & $\begin{array}{l}\text { El liderazgo no crea un trato } \\
\text { personalizado con los } \\
\text { seguidores }\end{array}$ \\
\hline
\end{tabular}

En esta tabla se presentan las dimensiones que conforman el constructo Liderazgo transformacional con sus interpretaciones según los resultados obtenidos.

Tabla 8

Dimensiones del constructo Liderazgo transaccional

\begin{tabular}{lll}
\hline Dimensión & Resultado de Análisis & Interpretación \\
\hline Conocimiento Contingente & Más del 90\% desfavorable & $\begin{array}{l}\text { No se evidencian recompensas } \\
\text { por el desempeño laboral. } \\
\text { Excepción Activa }\end{array}$ \\
Excepción Pasiva & Más del 95\% desfavorable & $\begin{array}{l}\text { No actúa ante desviaciones y } \\
\text { errores }\end{array}$ \\
Laissez Faire & Más del $90 \%$ desfavorable & $\begin{array}{l}\text { No interviene cuando ya se ha } \\
\text { dado el error }\end{array}$ \\
& & $\begin{array}{l}\text { No asume la responsabilidad } \\
\text { de líder, es un no liderazgo. }\end{array}$ \\
\hline
\end{tabular}

En esta tabla se presentan las dimensiones que conforman el constructo Liderazgo transaccional con sus interpretaciones según los resultados obtenidos. 


\section{Análisis descriptivo de la variable Autoeficacia emprendedora}

Se realizó un análisis de escala de Likert para agrupar las respuestas de las mencionadas escalas en cuatro nuevas categorías que van desde Muy desfavorable, desfavorable, favorable y Muy favorable.

\section{Tabla 9}

Dimensiones del constructo Autoeficacia Emprendedora

\begin{tabular}{lll}
\hline Dimensión & Resultado de Análisis & Interpretación \\
\hline $\begin{array}{l}\text { Afrontar cambios } \\
\text { inesperados }\end{array}$ & $\begin{array}{l}\text { Más del 50\% de la muestra } \\
\text { indicó una respuesta } \\
\text { desfavorable }\end{array}$ & $\begin{array}{l}\text { No se sienten capaces de trabajar } \\
\text { bajo incertidumbre y adaptarse a } \\
\text { cambios, desarrollando y dirigiendo } \\
\text { nuevos proyectos innovadores. }\end{array}$ \\
\hline $\begin{array}{l}\text { Construir un } \\
\text { entorno innovador }\end{array}$ & Más del 55\% favorable & $\begin{array}{l}\text { En su papel como directivos de la } \\
\text { comunidad son capaces de }\end{array}$ \\
& & $\begin{array}{l}\text { estimular la creatividad, iniciativa y } \\
\text { responsabilidad de las personas que } \\
\text { trabajan con ellos }\end{array}$ \\
\hline $\begin{array}{l}\text { Definir el objetivo } \\
\text { central del negocio }\end{array}$ & Más del 50\% favorable & $\begin{array}{l}\text { Pueden crear una visión para un } \\
\text { nuevo proyecto que une a los } \\
\text { miembros de la organización } \\
\text { alrededor de un objetivo común. }\end{array}$ \\
\hline $\begin{array}{l}\text { Desarrollar los } \\
\text { recursos humanos } \\
\text { clave }\end{array}$ & $\begin{array}{l}\text { Más del } 50 \% \text { de la muestra } \\
\text { indicó una respuesta } \\
\text { desfavorable }\end{array}$ & $\begin{array}{l}\text { Se dificulta atraer y retener } \\
\text { individuos que son claves en la } \\
\text { creación de un nuevo }\end{array}$ \\
\hline $\begin{array}{l}\text { Descubrir nuevos } \\
\text { productos y } \\
\text { oportunidades de } \\
\text { mercado }\end{array}$ & $\begin{array}{l}\text { Más del } 55 \% \text { de la muestra } \\
\text { indicó una respuesta } \\
\text { desfavorable }\end{array}$ & $\begin{array}{l}\text { Son incapaces de crear nuevos } \\
\text { productos o servicios que } \\
\text { satisfagan necesidades no cubiertas } \\
\text { de los clientes, permitiendo }\end{array}$ \\
$\begin{array}{l}\text { Iniciar relaciones } \\
\text { con inversores }\end{array}$ & $\begin{array}{l}\text { Más del 70\% de la muestra } \\
\text { indicó una respuesta } \\
\text { desfavorable }\end{array}$ & $\begin{array}{l}\text { Son incapaces de establecer } \\
\text { contactos que les permitan captar } \\
\text { los recursos necesarios para crear } \\
\text { su propia empresa. }\end{array}$ \\
\hline
\end{tabular}




\section{Análisis de correlación entre variables}

Tabla 10

Correlación Liderazgo Transformacional y Autoeficacia Emprendedora

\begin{tabular}{llll}
\hline & \multicolumn{1}{c}{$\begin{array}{c}\text { Liderazgo } \\
\text { transformacional }\end{array}$} & $\begin{array}{c}\text { Autoeficacia } \\
\text { emprendedora }\end{array}$ \\
\hline $\begin{array}{l}\text { Liderazgo } \\
\text { transformacional }\end{array}$ & $\begin{array}{c}\text { Correlación de } \\
\text { Pearson }\end{array}$ & 1 &, $777^{* *}$ \\
\cline { 2 - 3 } & Sig. (bilateral) &, 000 \\
\cline { 2 - 4 } $\begin{array}{l}\text { Autoeficacia } \\
\text { emprendedora }\end{array}$ & $\begin{array}{l}\text { Correlación de } \\
\text { Pearson }\end{array}$ &, $777^{* *}$ & 51 \\
\cline { 2 - 3 } & Sig. (bilateral) &, 000 & 1 \\
\hline **. La correlación es significativa al nivel 0,01 (bilateral). & \\
\hline
\end{tabular}

Se puede evidenciar que existe una correlación significativa al 0,01 (bilateral) entre la variable Liderazgo transformacional y Autoeficacia emprendedora.

\section{Tabla 11}

Correlación Liderazgo Transaccional y Autoeficacia Emprendedora

\begin{tabular}{lllc}
\hline & & $\begin{array}{c}\text { Autoeficacia } \\
\text { emprendedora }\end{array}$ & $\begin{array}{c}\text { Liderazgo } \\
\text { transaccional }\end{array}$ \\
\hline \multirow{2}{*}{ Autoeficacia emprendedora } & Correlación de Pearson & 1 &, 250 \\
\cline { 2 - 4 } & Sig. (bilateral) & &, 077 \\
\cline { 2 - 4 } & $\mathrm{N}$ & 51 & 51 \\
\hline Liderazgo transaccional & Correlación de Pearson &, 250 & 1 \\
\cline { 2 - 4 } & Sig. (bilateral) &, 077 & 51 \\
\cline { 2 - 4 } & $\mathrm{N}$ & 51 & \\
\hline
\end{tabular}

Los resultados indicaron que no existe una relación significativa entre la variable Liderazgo transaccional y Autoeficacia emprendedora.

\section{Conclusiones}

La muestra analizada indicó que los emprendedores de la comunidad en su mayoría son mujeres, de la misma manera, se evidencia una edad que refiere a una población adulta joven, la mayoría de los emprendedores han alcanzado el nivel secundario de educación y son de 
nacionalidades indígenas de la Amazonía ecuatoriana, este resultado podría inferir (sujeto a futuros análisis) que los emprendimientos obedecen hacia actitudes de necesidad antes que por oportunidad y también que la educación formal no representa una limitante para desarrollar emprendimientos. El análisis descriptivo de la variable liderazgo transformacional y transaccional indicó resultados desfavorables para cada una de las dimensiones, este resultado se interpreta como una falta de desarrollo de liderazgo en los emprendedores del sector turístico de la comunidad amazónica, sin embargo, se debe considerar como lo han indicado otras investigaciones que el liderazgo tradicional en las comunidades, se vuelve débil, normalmente porque es temporal y fragmentario, el cual es repartido entre familias, bandas, linajes y varias asociaciones. Con respecto a la variable autoeficacia emprendedora se evidencia respuestas favorables para algunas dimensiones, como definir el objetivo central del negocio y construir un entorno innovador sin embargo, el resto de dimensiones se determina un comportamiento desfavorable, se concluye que las creencias en las habilidades emprendedoras no son lo suficientes, se puede atribuir a varios factores tanto internos como externos como la edad, nivel de educación, ambiente de trabajo, etc. Lo cual estaría dentro de un campo amplio de futuras investigaciones. El análisis correlacional indicó una relación significativa entre Liderazgo transformacional y Autoeficacia emprendedora, sin embargo, no se evidencia una correlación entre Liderazgo transaccional y la Autoeficacia emprendedora, con estos resultados se concluye que existe una predisposición de los emprendedores hacia un liderazgo que integra la motivación, compromiso, moral en sus actividades emprendedoras y se limita el concepto y actitud de transacción al desarrollar emprendimientos, es decir, se evidencia una fuerte influencia del crecimiento transformador y de cambio en la comunidad antes que el sentido de recompensa por cada acción realizada, esta investigación abre nuevas líneas de investigación para conocer el comportamiento emprendedor en comunidades indígenas de la Amazonía ecuatoriana, de regiones y contextos similares, de la misma manera, se puede realizar investigaciones que determinen causalidades entre las variables analizadas, en la cual se puede integrar constructos como cultura y género.

\section{Referencias bibliográficas}

Alvarez,C.,Noguera,M., \& Urbano,D.(2012).Condiciones del entorno y emprendimiento femenino: Un estudio cuantitativo en España. Economía industrial.

Antares, A. (2013). Interdependencia entre Liderazgo Transformacional, cultura organizacional y cambio educativo. Revisata Iberoamericana sobre Calidad, Eficacia y cambio en educación, $11(1)$.

Obtenido Hhttp://www.rinace.net/reice/numeros/arts/vol11num1/art5.pdf

Avolio,B., Bass,B., \& Jung, D. (1999). Re-examining the components of transformational and transactional leadership using the Multi-factor Leadership Questionnaire. Journal of Occupational and Organizational Psychology, 441-462.

Bass,B.,\& Avolio,B. (1994). Improving Organizational Effectiveness through Transformational Leadership. London-New Delhi: Thousand Oaks.

Bass, B., \& Avolio, B. (2000). MLQ Multifactor Leadership. Mind Garden.

Burns, J. (1978). Leadership. New York: Harper \& Row.

Contreras,F., \& Barbosa,D. (2013). Del liderazgo transaccional al liderazgo transformacional: implicaciones para el cambio organizacional. Revista Virtual Universidad Católica del

Esta obra se comparte bajo la licencia Creative Common Atribución-No Comercial 4.0 International (CC BY-NC 4.0) Revista de la Universidad Internacional del Ecuador. URL: https://www.uide.edu.ec/ 
Norte(39),

152-164.

Obtenido

de

http://revistavirtual.ucn.edu.co/index.php/RevistaUCN/article/view/433/886

Corporate Excellente. (2014). Por un liderazgo basado en valores y en la calidad humana: cuando ética y eficacia avanzan juntos. En C. C. forReputationLeadership. Madrid.

Cruz-Ortiz,V.,Salanova, M., \& Martínez , I. (2013). Liderazgo transformacional y desempeño grupal: unidos por el engagement grupal. Revista de Psicología Social(28), 183-196. doi:Doi: 10.1174/021347413806196762

Durán,M., \& Castañeda,D.(2015). Relacion entre liderazgo Transfromacional y Transaccional con la conducta de compartir conocimientos en dos empresas de servicios. Universidad católica de Colombia, 18(1), 135-147. Obtenido de http://www.dx.doi.org/10.14718/ACP.2015.18.1.13

Durán, E., \& Arias, D. (2016). Actitud emprendedora y estilos emocionales. Contribuciones para el diseño de la formación de futuros emprendedores. Gestión de la Educacón, 6(2).

Fernández,M., \& Quintero,N.(2017). Liderazgo transformacional y transaccional en emprendedores venezolanos. Revista Venezolana de Gerencia, 56-74.

Gómez , G., \& Ezama, P. (2012). Metodología Delphi como técnica de estudio de la validez de contenido. Anales de Psicología, 1011-1020.

Gómez Díaz, R., Quero Gervilla, M., López Gómez, E., Medina Domínguez, M. C., \& Pérez Navío, E. (26 Noviembre 2016). Formación de líderes: Identificación de funciones y competencias en los centros educativos y en las organizaciones coperativas. En J. Bernal Agudo (Ed.), Globalización y organizaciones Educativas. Simposio llevado a cabo en el XIV CONGRESO INTERUNIVERSITARIO DE ORGANIZACIÓN DE INSTITUCIONES EDUCATIVAS (CIOIE). Zaragoza.

Guanilo, W. (2017). El Liderazgo Transaccional y su relación con el Desempeño Laboral en la empresa Conservas Ricofres, Chancay - 2017 (Tesis de Pregrado). Universidad César Vallejo, Lima, Peru.

Hermosilla, D., Amutio, A., Da Costa, S., \& Páez, D. (2016). El Liderazgo transformacional en las organizaciones: variables mediadoras y consecuencias a largo plazo. Scielo - Revista de Psicología del Trabajo y de las Organizaciones, 32(3). doi:http://dx.doi.org/10.1016/j.rpto.2016.06.003

Ibarra,E., Tandazo,A., Alemán,R., Bravo, C., \& Caballero, E. (s.f.). El agroturismo impulsado por "Vitalideas" como alternativa de desarrollo sostenible en la amazonia ecuatoriana: Estudio de caso "Las Cabañas". III Jornada Iberoamericana en saludo al día mundial del medio ambiente - Ecuador 2017. Puyo: Universidad Estatal Amazónica.

Lasio,V., Caicedo,G., Ordeñana,X., Samaniego,A., \& Zambrano,J. (2016). Global Entrepreneurship Monitor. Graduate School of Management, págs. 9-16.

Ledesma Almache, G. (2016). Estudio del comercio justo y su incidencia en el proyecto de emprendimiento de la Asociación indígena Kallari . Obtenido de UNIVERSIDAD INTERNACIONAL SEK: http://repositorio.uisek.edu.ec/bitstream/123456789/2180/1/PROYECTO\%20DE\%20FIN \%20DE\%20CARRERA.pdf

López, M., \& Sicilia, M. (2015). Identifcación de líderes de opinión leales en Twitter. Red de Revistas Científicas de América Latina y el Caribe, España y Portugal, 17(1), 108- 109. doi:10.5295/cdg.140508ml 
López, S., Flores, A., \& Bojórquez, A. (2016). El impacto de la autoeficacia emprendedora en los resultados de innovación de los emprendedores del sector de software en Yucatán. Red Internacional de Investigadores en Competitividad, 10.

Maldonado, J., Castro, F., \& Franco , L. (2017). Estudio de la inteligencia y habilidades emprendedoras en líderes políticos y sociales: Casos de éxito en Extremadura. Rassegna, 27(2), 57-64.

Manosalvas , L. (2017). Relación de la Inteligencia Emocional con la Intención del Emprendimiento y la Autoeficacia emprendedora (Tesis de Postgrado). Surco, Perú.

Marín, H., Manjarez, N., Ortega, V., Sablón, N., \& Romero, E. (2017). Desafíos de la gestión de talento humano en la actividad turística en la Amazonía Ecuatoriana. III Jornada Iberoamericana en saludo al día mundial del Medio Ambiente- Ecuador 2017( Universidad Estatal Amazónica).

Mendoza, A., \& Prideaux, B. (2017). Assessing ecotourism in an Indigenous community: using, testing and proving the wheel of empowerment framework as a measurement tool. Journal of Sustainable Tourism, 2(26), 1-2. Obtenido de https://www.tandfonline.com/doi/full/10.1080/09669582.2017.1347176?scroll=top\&need Access $=$ true

Molero, F., Recio, P., \& Cuadrado, I. (2010). LA MEDICIÓN DEL LIDERAZGO TRANSFORMACIONAL Y TRANSACCIONAL EN ESPAÑA A TRAVÉS DEL MLQ. Obtenido de infocop online: http://www.infocop.es/view_article.asp?id=3053

Muller, P. (2016). Reputation, trust and the dynamics of leadership in communities of practice. Journal of Management \& Governance, 10(4), 381-400. doi:DOI 10.1007/s10997-0069007-0

Munch, L. (2011). Liderazgo y Direcciòn. En El liderazgo del siglo XXI (pág. 146). Mexico: Trillas S.A.

Murillo, G., González, C., \& Perdomo, G. (2010). Gestión, poder, innovación y estrategia en las organizaciones.

Naranjo, R. (2015). Habilidades gerenciales en los líderes de las medianas empresas de Colombia. Revista científica Pensamiento y Gestión(38), 38. Obtenido de http://rcientificas.uninorte.edu.co/index.php/pensamiento/article/view/7703

Nielsen, K. (2014). Leadership and Climate in a Psychologically Healthy Workplace. In Day, Kelloway, \& Hurrell (Eds.). Workplace Well-Being Building Positive \& Psychologically Healthy Workplaces, 226-244.

Olvera, J., Llorens, S., Acosta, H., \& Salanova, M. (2017). El liderazgo transformacional y la confianza como antecedentes del desempeño en equipo en el ámbito sanitario. ( $\mathrm{S}$. d. Universidad de Murcia, Ed.) Repositori Universitat Jaume I, 33(2), 365-375. doi:http://dx.doi.org/10.6018/analesps.33.2.237291

Orozco Dussán, L. (2014). Liderazgo y emprendimiento. Factores culturales relacionados. Obtenido de Universidad del Rosario: http://repository.urosario.edu.co/flexpaper/handle/10336/10099/10184550442014.pdf? sequence $=1 \&$ isAllowed $=\mathrm{y}$

Palma, Y. (2011). Factores que inciden en la intencionalidad de emprender de los estudiantes universitarios en Panamá (Tesis Doctoral). España: Universidad Politécnica de Cataluña.

Peredo, B., Ordóñez, A., \& Belohrad, V. (2015). Past and Present Perspectives on Indigenous Tourism in the Pastaza Province of Ecuador: The Case of Kapawi. The International Indigenous Policy Journal, 6(4). doi:10.18584/iipj.2015.6.4.7 
Pérez, C., Fernández, J., Ubierna, F., \& Arranz, N. (2016). El desarrollo de la motivación emprendedora en estudiantes de turismo. Un análisis comparado entre alumnos de grado y postgrado. Esic Market Economics and Business Journal, 47(1), 67-93. doi:DOI: 10.7200/esicm.153.0471.2e

Peters, M. (2005). Entrepreneurial skills in leadership adn human resource management evaluated by apprentices in small tourism business. Education \& Training, 8(47), 575-591.

Salazar, T., Vargas, J., \& Salazar, J. (2017). Liderazgo y decisión en el emprendimiento de una organización para su crecimiento en periodo de crisis. En ESTRATEGIAS E INTERACCIONES PARA LA GESTIÓN DEL DESARROLLO SUSTENTABLE, LA CALIDAD DE VIDA, RESPONSABILIDAD EMPRESARIAL Y TRANSFERENCIA DEL CONOCIMIENTO EN LAS ORGANIZACIONES (págs. 187-189). México: Competitive Press, S.A. de C.V.

Sopó, G., Salazar, M., Guzmán, E., \& Vera, L. (2017). Liderazgo como competencia emprendedora. Espacios, 24.

Spinelli, S., \& Adams, R. (2012). New Venture Creation.

Torres, J. (2015). Liderazgo comarcal: una estructura y herramienta para la descentralización en las comunidades de las Comarcas Malacatoya, La Concepción 1, Boaco, 2015. Revista Senderos Universitarios(3), 22-31.

Vadillo Bueno, S. (2011). Empresas Exitosas, Personas Exitosas. En Secretos de la empresa triunfadora (pág. 85). Mexico: Trillas S.A.

Varela, H. (2010). Liderazgo transaccional vs Liderazgo Transformacional. Obtenido de Blog: Pensamiento Imaginativo: http://manuelgross.bligoo.com/content/view/785903/

Whiteford, L., \& Padros, C. (2015). Community Participatory Involvement: A Sustainable Model for Global Public Health. Anthropology book Forum.

$\mathrm{Xu}$, L. (2014). A Tale of Trinity in Founderes Identity: The Case of New Venture Creation. Journal of Management, 15(5). 